\title{
Lossy Image Compression Using PCA and Contourlet Transform
}

\author{
Yaxiong Chen, Zhangcan HUANG,Hao Sun, Mengying Chen, Hua Tan \\ School of Science, Wuhan University of Technology, Wuhan 430070, China
}

\begin{abstract}
With the rapid development of Internet, image information is growing. It requires a lot of image storage and transmission. In order to reduce the storage and get better image quality, image compression algorithm is studied. The paper proposes a new image compression algorithm that combines principal component analysis (PCA) and Contourlet Transform (CT). Because PCA has good image quality, but the compression ratio is low, and CT compression algorithm has high compression ratio and good PNSR value. The image is decomposed by PCA. The image data is divided into blocks, and each block is used as a sample vector, then select covariance matrix of $\mathrm{k}$ larger eigenvalues corresponding eigenvector to realize image compression. Then the image is compressed again using CT compression algorithm. Compared with the results of JEPG2000 and CT compression algorithm, the results show that the proposed algorithm has better performance than JEPG2000 and CT compression algorithm. In the same compression ratio, PNSR value of proposed algorithm is about $3 \mathrm{~dB}$ higher than that of JEPG2000, and 2dB higher than that of CT compression algorithm.
\end{abstract}

\section{Introduction}

With the rapid development of Internet, image information is growing. It requires a lot of image storage and transmission [1]. The amount of information in the digital image is very large, and it occupies a large amount of resources. So it is important to use appropriate method to compress the image in order to store and transmit. Image compression is divided into lossless compression and lossy compression [2-3]. Principal component analysis algorithm is a kind of feature vector based lossy compression algorithm, which has many applications in image compression and digital watermarking [4-8]. Principal component analysis converts the data set into a low dimensional feature space, and retains most of the original data information content [9]. Contourlet transform are used in the image denoising [10], image fusion [11] and image enhancement [12], and the effect is good. CT compression algorithm is to make full use of contourlet transform to decompose the image with multiscale and multidirection [13], despite contourlet transform can produce 4 / 3 redundancy, but the use of hard threshold of coefficients processing and run length coding with redundancy is eliminated and realize image compression [14]. JPEG2000 is a new generation of still image compression standard, it uses the wavelet transform, the optimized truncation embedded block encoding and efficient code stream organization form [15-16].In this paper, a new image compression algorithm is proposed, which combines PCA and contourlet transform. CT compression algorithm is to first convert by contourlet transform, using hard threshold for contourlet transform coefficients, treatment with run length coding for image compression. Because PCA has good image quality, but the compression ratio is low, and CT compression algorithm has high compression ratio and good PNSR value. The proposed algorithm has been tested in the famous images such as airfield, Lena, etc., and has been compared with JEPG2000 and CT compression algorithm.

\section{Image compression based on principal component analysis and Contourlet transform}

\subsection{Principal component analysis of image compression}

Principal component analysis is a kind of exploratory statistical analysis method, which will be distributed on a set of variables to focus on a few principal components. Principal component analysis is a kind of non-supervised method, because it does not need to output information.

Assume a sample set $X$, contains $m$ samples, the dimension of each sample is $n$,

$$
\begin{aligned}
& X=\left\{x_{1}, x_{2}, \ldots, x_{m}\right\} \\
& x_{i}=\left(x_{i 1}, x_{i 2}, \ldots, x_{i n}\right) \in R^{n}
\end{aligned}
$$

The sample set $X$ is organized into a sample matrix, each row of which is a sample, and each column is a dimension, $S$ is $m * n$ matrix. 


$$
\begin{aligned}
& \hat{X}_{i}=X_{i}-\bar{X} \\
& \bar{X}=\frac{1}{m} \sum_{i=1}^{m} X_{i}
\end{aligned}
$$

The sample matrix of $\hat{X}_{i}$ is denoted as $\hat{S} \in R^{m \times n}$, then the covariance matrix of $S$ is $C$, which is known by the formula (1).

$C=\frac{1}{m} \hat{S}^{T} \hat{S}=\frac{1}{m} \sum_{i}^{m} \hat{X}_{i}^{T} \hat{X}_{i}$

According to matrix theory, we know that there exists an orthogonal matrix $P, P^{T} C P=A$. For the specific operation of the orthogonal matrix $P$ : The $\mathrm{C}$ eigenvalue decomposition, get the eigenvalue matrix as a feature vector, and the orthogonal matrix is denoted as $P$. Obviously, $P, A \in R^{n^{*} n}$. To take the maximum of the former $\mathrm{K}$ eigenvalues, then the $\mathrm{K}$ feature values of the $A_{1}$ feature vectors are composed of new feature vector matrix, which is the principal component of PCA transform.

Because of $P_{l}^{T} C P_{I}=A_{l}$, it can be obtained

$$
\begin{aligned}
& P^{T}\left(\frac{\hat{S}^{T} \hat{S}}{m}\right) P=\frac{(\hat{S} P)^{T} \hat{S} P}{m}=A \\
& \frac{\left(\hat{S} P_{1}\right)^{T} \hat{S} P_{1}}{m}=A_{1}
\end{aligned}
$$

Then $S_{1}=\hat{S} P_{1}$, the formula (4) deformation is

$$
\frac{1}{m} S_{1}^{T} S_{1}=A_{1}
$$$$
\hat{S}=S_{1} P_{1}^{T}
$$

PCA is used to image compression and block image data, each block as a sample vector, and then calculate corresponding feature vector based on the $\mathrm{k}$ eigenvalues of covariance matrix $C$, which is main component of PCA, and give up the corresponding feature vector of the smaller $n-k$ eigenvalues, which constitute the transformation matrix $P_{l}$. The center of the sample matrix $\hat{S}$ after transformation to get $S_{l}$, the compression ratio is $n: k$, The formula (6) can reconstruct the original image, and the mean square error is equal to the sum of $n-k$ eigenvalues.

\subsection{Contourlet transform of image compression}

Contourlet Transform is the use of Laplacian pyramid decomposition(LP)and directional filter banks(DBF) to achieve a multiresolution, local and multi direction image representation method. Firstly, the image is decomposed by using LP to capture the singular points, and then the DFB is used to connect the singular points of the similar direction. LP is a low pass filter for the original image, and then the low frequency image is sampled and filtered, The difference between the original image and the predicted image is Laplasse's decomposition. The low pass image continues to carry on the Laplasse decomposition, and finally completes the multi scale decomposition of the image. In the reconstruction, the low pass image is sampled and filtered with a band-pass image to complete the reconstruction, the transformation process is performed on the low frequency image, and the image is reconstructed. After the image is decomposed by Laplasse, the singular points of the close direction of the image are connected to the linear structure, so that the image can be decomposed by using a binary tree decomposition. The band pass image is divided into $2^{\mathrm{L}}$ wedge frequency sub band. The wedge frequency spectrum is obtained by using the combination of Quincunx Bank and DFB.

Because of the structure of the contourlet transformation, the support set is the "long form" structure which is transformed with the scale, so that the image can be decomposed completely. Image Peppers can be carried out by contourlet transform.

\section{Encoding}

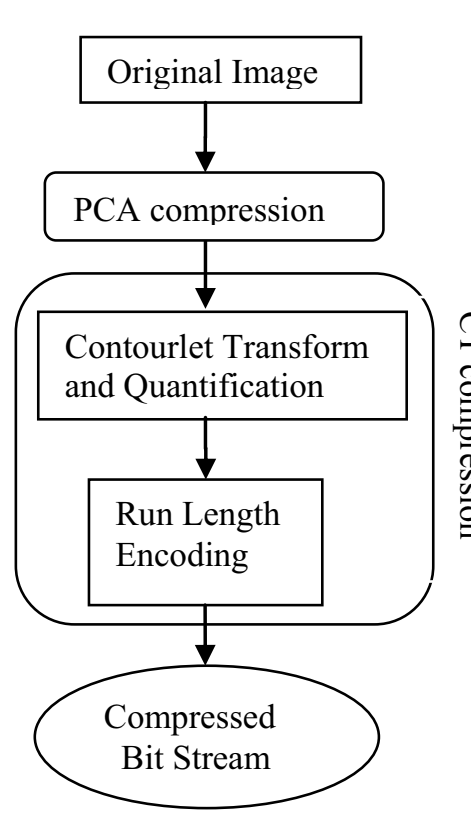

\section{Decoding}

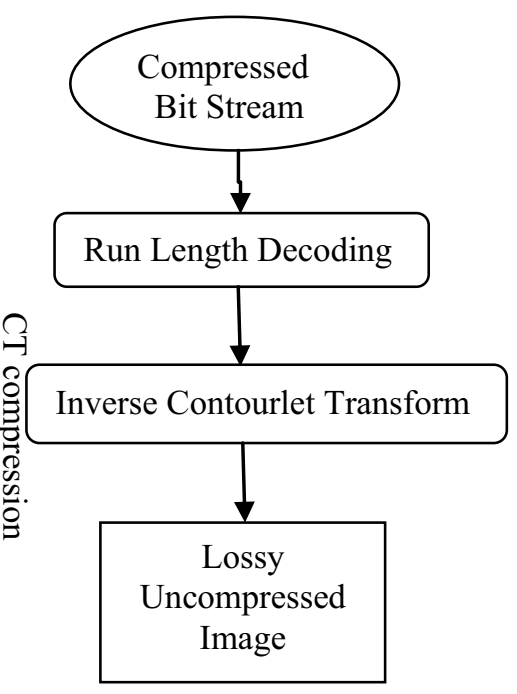

Figure 1. Block diagram of the proposed image compression algorithm

Secondly, Contourlet transform has a large number of coefficients, which represent the direction information and texture information. Large coefficients contain more information, more important than small coefficients. With the decomposition of the scale, the Contourlet coefficient decays rapidly. By the decomposition, the fine scale contains a large number of small coefficients, these small coefficients around 0 floating. This paper uses a simple hard threshold processing to remove the small coefficient of the decomposition. While retaining the high frequency sub bands in the larger coefficient, so that the image quality can be guaranteed for the follow-up of the compression process. As shown below:

$$
g(x, y)=\left\{\begin{array}{c}
f(x, y),|f(x, y)|>\beta \\
0,|f(x, y)| \leq \beta
\end{array}\right.
$$

Among them, $g(x, y)$ indicates that the coefficients are processed by threshold, $f(x, y)$ represents the change coefficient before processing, $\beta$ represents setting the 
threshold, the greater the $\beta$, the greater the compression ratio.

Finally, using the run-length coding of quantitative coefficients are encoded.

\subsection{Algorithm of image compression}

In order to ensure the compression quality and improve the compression ratio, this paper combines PCA and Contourlet transform, and the image is compressed by two levels. Specific algorithms are shown in figure 1.

The image data is divided into blocks, and each block is used as a sample vector, then select covariance matrix of $\mathrm{k}$ larger eigenvalues corresponding eigenvector to realize the first-stage compression of the image. Reconstruction of image uses contourlet transform. Contourlet transform coefficients are hard threshold processing. To achieve image compression using run length encoding in entropy coding.

Reconstruction of image data based on PCA and contourlet transform. Run length decoding of compressed results are run in contourlet inverse transformation. The image is reconstructed.

\section{Experiments and discussions}

In order to verify the effectiveness of the proposed method, 5 images of the compression experiments were carried out. All images are 512*512 gray-scale images. According to the literature, contourlet transform using '97 ' pyramid decomposition and 'pkva' direction filter 3 layer decomposition, several layers direction is $[1,2,3]$. By changing the contribution of the singular value of the image signal and the contourlet coefficient quantization threshold, the reconstructed images are obtained under different compression ratios, and the PNSR and SSIM are calculated.

Table 1. PNSR values in $\mathrm{dB}$ for 20:1 compression.

\begin{tabular}{cccc}
\hline Image/Method & CT & JEPG2000 & Proposed \\
\hline airfield & 30.46 & 27.32 & 31.63 \\
Goldhill & 31.96 & 32.18 & 34.19 \\
Lena & 34.16 & 35.99 & 36.64 \\
Peppers & 33.89 & 35.07 & 36.03 \\
Boats & 32.57 & 33.18 & 35.13 \\
\hline
\end{tabular}

Table 2. PNSR values in $\mathrm{dB}$ for 40:1 compression.

\begin{tabular}{cccc}
\hline Image/Method & CT & JEPG2000 & Proposed \\
\hline airfield & 30.17 & 24.88 & 31.06 \\
Goldhill & 31.52 & 29.72 & 33.33 \\
Lena & 33.12 & 32.75 & 35.88 \\
Peppers & 32.95 & 32.4 & 35.5 \\
Boats & 31.87 & 29.76 & 33.5 \\
\hline
\end{tabular}

Table 1 shows the comparison PNSR value of the proposed algorithm, JEPG 2000 and CT compression algorithm in the 20:1 compression ratio. And the Table 2 and Table 3 respectively present the PNSR values which the compression ratios are 40:1 and 80:1. According to the Table 1, Table 2 and Table 3, it can be found that the proposed algorithm is the best one of the three algorithms.

Table 3. PNSR values in $\mathrm{dB}$ for $80: 1$ compression.

\begin{tabular}{cccc}
\hline Image/Method & CT & JEPG2000 & Proposed \\
\hline airfield & 27.95 & 22.64 & 30.87 \\
Goldhill & 28.53 & 27.69 & 32.67 \\
Lena & 29.01 & 29.62 & 34.87 \\
Peppers & 29.33 & 29.54 & 34.6 \\
Boats & 28.7978 & 26.76 & 33.31 \\
\hline
\end{tabular}

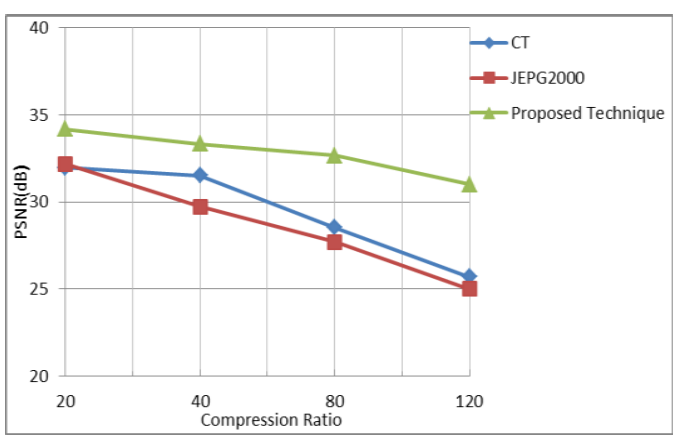

Figure 2. Comparison of PNSR values of peppers image for different compression ratios for the proposed algorithm, JEPG2000 and Contourlet transform.

Under the same compression ratio, the PNSR value of proposed algorithm is 4dB higher than that of JEPG2000, and $2 \mathrm{~dB}$ higher than that of CT compression algorithm. Besides, Table 4, Table 5 and Table 6 respectively show the SSIM values in the compression ratio of 20:1, 40:1 and $80: 1$.

Table 4. SSIM comparison for 20:1 compression.

\begin{tabular}{cccc}
\hline Image/Method & CT & JEPG2000 & Proposed \\
\hline airfield & 0.5546 & 0.698 & 0.74 \\
Goldhill & 0.6541 & 0.839 & 0.8312 \\
Lena & 0.8057 & 0.91 & 0.9057 \\
Peppers & 0.7933 & 0.875 & 0.876 \\
Boats & 0.7515 & 0.898 & 0.894 \\
\hline
\end{tabular}

Table 5. SSIM comparison for 40:1 compression.

\begin{tabular}{cccc}
\hline Image/Method & CT & JEPG2000 & Proposed \\
\hline airfield & 0.4758 & 0.606 & 0.6409 \\
Goldhill & 0.598 & 0.752 & 0.7733 \\
Lena & 0.738 & 0.868 & 0.8838 \\
Peppers & 0.7399 & 0.839 & 0.8581 \\
Boats & 0.672 & 0.828 & 0.812 \\
\hline
\end{tabular}

Obviously, the proposed algorithm is similar to JEPG2000 under the compression ratio of 20:1. it can be seen that the proposed algorithm is much better than the others under the compression ratio of 40:1 and 80:1. With the increasing of the compression ratio, the proposed algorithm is better. 
Table 6. SSIM comparison for 80:1 compression.

\begin{tabular}{cccc}
\hline Image/Method & CT & JEPG2000 & Proposed \\
\hline airfield & 0.3392 & 0.52 & 0.6117 \\
Goldhill & 0.4442 & 0.67 & 0.7089 \\
Lena & 0.5322 & 0.808 & 0.8375 \\
Peppers & 0.5642 & 0.783 & 0.823 \\
Boats & 0.5145 & 0.74 & 0.8021 \\
\hline
\end{tabular}

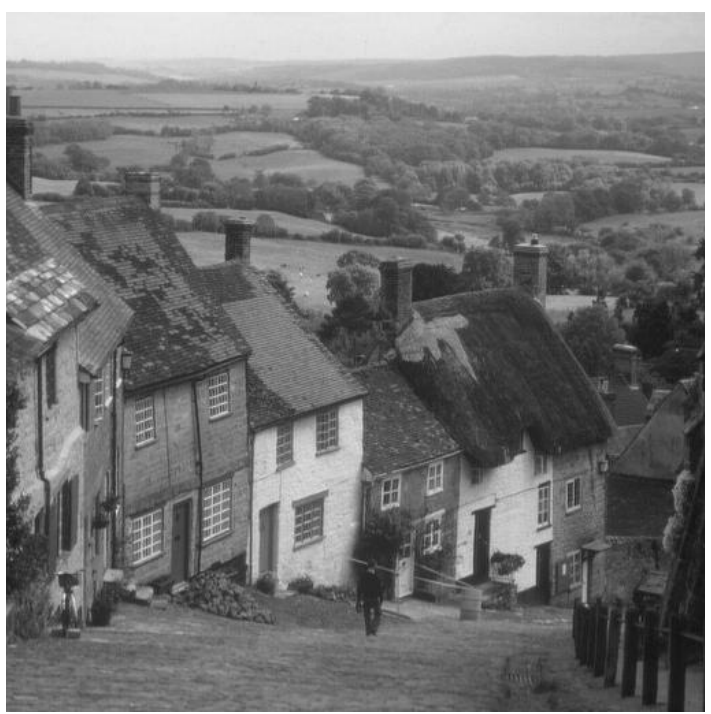

(a)

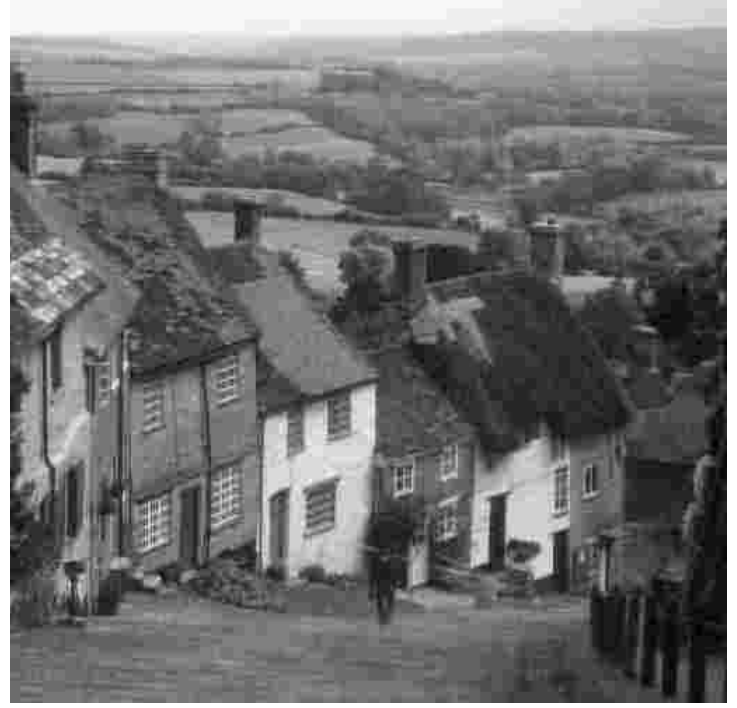

(c) compression ratio. Because of the large eigenvectors contribution to the image signal, PCA has good image quality, so it can be combined with CT compression algorithm to improve the image quality and SSIM value in the same compression ratio.

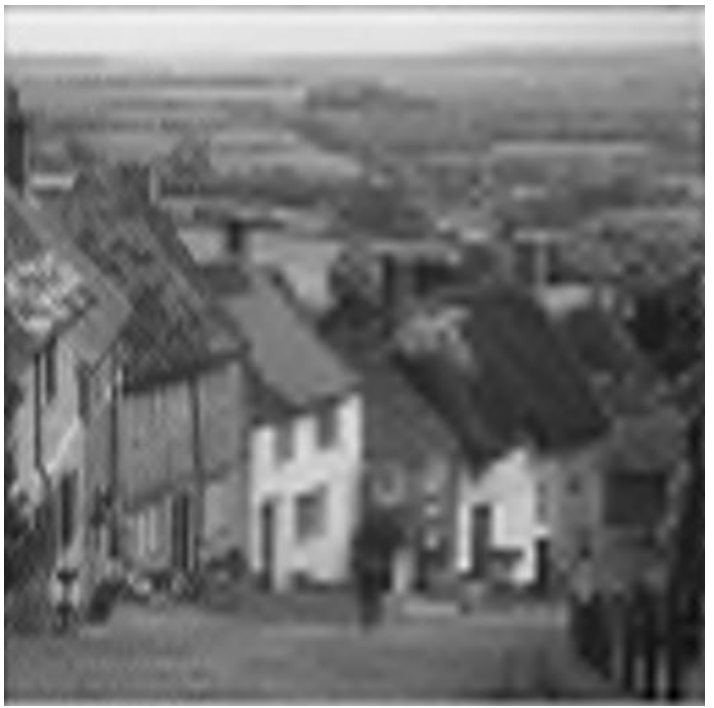

(b)

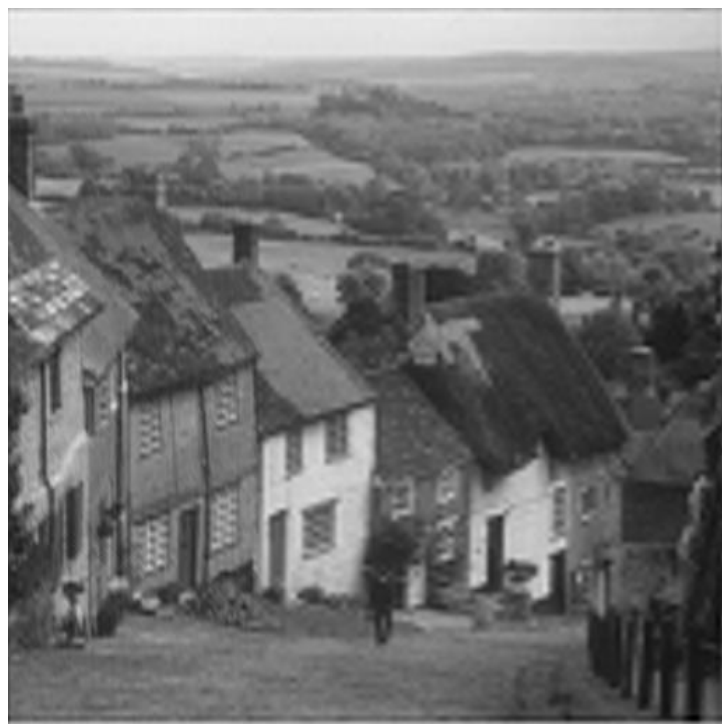

(d)

Figure 3. Original Goldhill image, and compressed image by using(b)Contourlet transform, (c)JEPG2000, and(d) proposed algorithm at 40:1 compression ratio.

Figure 2 shows that the PNSR value of the proposed algorithm is higher than the other algorithms, when the compression ratio become more higher, the proposed algorithm is better than the other two algorithms.

Figure 3 shows that image Goldhill is compressed by three methods in the compression ratio of 40:1. Overall, quantitative and qualitative results show that the proposed algorithm has better image quality and

\section{Conclusions}

In this paper, a new lossy compression algorithm is proposed, which combines PCA and Contourlet transform. The proposed algorithm uses PCA to ignore the smaller eigenvectors to reconstruct the compressed image. This image was compressed again by using CT compression algorithm. The advantages of PCA and 
Contourlet transform are effectively combined. The compression ratio of proposed algorithm is the product of the compression ratio of PCA and the compression ratio of CT compression algorithm. The result of proposed algorithm is obviously better than JEPG2000 and CT compression algorithm, which has a certain application prospect.

\section{References}

1. An H, Meng L, Zhao L, et al, Long-distance Transmission and High-speed and Real-time Storage Technology of Image Data, J. Video Engineering. 37(3) (2013) 175-178.

2. Groach M, Garg D A, DCSPIHT: Image compression algorithm, J. International Journal of Engineering Research and Applications. 18(2) (2012) 560-567.

3. Rufai A M, Anbarjafari G, Demirel H, Lossy medical image compression using Human coding and singular value decomposition (Signal Processing and Communications Applications Conference, 2013).

4. Du, Qian, and James E. Fowler. "Hyperspectral image compression using JPEG2000 and principal component analysis." IEEE Geoscience and Remote Sensing Letters, 4.2 (2007): 201-205.

5. Ting, Lim Sin, David Yap Fook Weng, and Nurulfajar Bin Abdul Manap. "A Novel Approach for Arbitrary-Shape ROI Compression of Medical Images Using Principal Component Analysis (PCA)." Trends in Applied Sciences Research, 10.1 (2015): 68.

6. Du, Qian, et al. "Hyperspectral image compression and target detection using nonlinear principal component analysis." in Proc. Satell. Data Compression Commun. Process. IX, 2013, vol. 8871

7. Shi, Qiuyan, Xingsong Hou, and Xueming Qian. "Hyperspectral image compression based on DLWT and PCA." Proceedings of the 7th ACM International Conference on Internet Multimedia Computing and Service, 2015.

8. Saboori, Arash, and S. Abolfazl Hosseini. "A new method for digital watermarking based on combination of DCT and PCA." IEEE Telecommunications Forum Telfor (TELFOR), 2014.

9. Wang, Chih-Wen, and Jyh-Horng Jeng. "Image compression using PCA with clustering." IEEE International Symposium on Intelligent Signal Processing and Communications Systems (ISPACS), 2012.

10. Min, Dong, Zhang Jiuwen, and Ma Yide. "Image denoising via bivariate shrinkage function based on a new structure of dual contourlet transform."Signal Processing, 109 (2015): 25-37.

11. Hou C, Yang M S, Zhang G D, Image Enhancement Method Based on Wavelet-Contourlet Transform Image Fusion of Intelligent Monitoring, J. Control Engineering of China. 21(1) (2014) 62.

12. BAI, Chun-xia, and Wen-zhong ZHAO. "Treatment of multi-dimensional signal based on complex wavelet-contourlet transform [J]." Automation \& Instrumentation, 1 (2011): 044.

13. Do, Minh N., and Martin Vetterli. "The contourlet transform: an efficient directional multiresolution image representation." IEEE Transactions on Image Processing, 14.12 (2005): 2091-2106.

14. Jian Liu, Yinglei Cheng, Qiang Xu. SAR image compression algorithm based on Contourlet transform $[\mathrm{J}]$. Science and technology and Engineering, 12 (2012):7252-7255.

15. Grosbois, Raphael, Diego Santa-Cruz, and Touradj Ebrahimi. "New approach to JPEG 2000 compliant region of interest coding." SPIE International Symposium on Optical Science and Technology,2001.

16. Christopoulos, Charilaos, Athanassios Skodras, and Touradj Ebrahimi. "The JPEG2000 still image coding system: an overview." IEEE Transactions on Consumer Electronics, 46.4 (2000): 1103-1127. 